\title{
Mental Health Challenges and Psycho-social Interventions amid COVID-19 Pandemic: A Call to Action for Pakistan
}

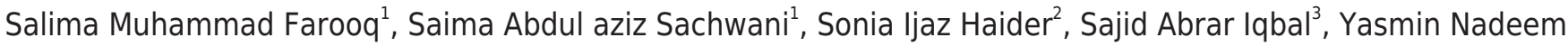 \\ Parpio $^{1}$ and Humera Saeed ${ }^{4}$ \\ ${ }^{1}$ School of Nursing and Midwifery, Aga Khan University, Karachi, Pakistan \\ ${ }^{2}$ Department for Educational Development, Aga Khan University, Karachi, Pakistan \\ ${ }^{3}$ Aga Khan University, Karachi, Pakistan; School of Nursing, University of Faisalabad, Pakistan \\ ${ }^{4}$ Department of Psychiatry, Aga Khan University, Karachi, Pakistan
}

\begin{abstract}
The increase in death and spread-related coronavirus (COVID-19) has shifted the world focus to the containment of the disease by emphasising measures to prevent spread in the general population. Such a complex, threatening, and unprecedented situation has left the psycho-social wellbeing needs of general public unaddressed. This paper aims to review the current COVID-19 scenario and its effects on the psycho-social wellbeing of people; and an attempt to shed some light on the aforementioned questions. Furthermore, the review will propose some recommendations for overcoming the mental illness issues, during and after the COVID-19 outbreak. We extracted information from reliable published international and_national literature and reviewed anecdotes from media content from January to June 2020. The mental health implications of this outbreak will be longlasting; however, by prioritising, investing diligently, and taking a collective approach, this challenge can be dealt with in a promising manner.
\end{abstract}

Key Words: Mental health, Psycho-social interventions, COVID-19, Pakistan.

How to cite this article: Farooq SM, Sachwani SAA, Haider SI, lqbal SA, Parpio YN, Saeed H. Mental Health Challenges and Psycho-social Interventions amid COVID-19 Pandemic: A Call to Action for Pakistan. J Coll Physicians Surg Pak 2020; 30(JCPSPCR):CR59-CR62 https://doi.org/10.29271/jcpsp.2020.JCPSPCR.CR59.

The global pandemic of noval coronavirus (COVID-19) has wrecked the health and economy of the world. In Pakistan, more than 200,000 cases have been reported so far and 4,000 people have died. As the disease is spreading rapidly, it has caused panic and fear among people of all age groups. Moreover, this crisis has also caused psychological distress because of its contagious nature, absence of definitive treatment, and the physical isolation that has been forced upon billions of people. The extensive news coverage on the pandemic has precipitated phobia of the disease and death, and created further uncertainty. COVID-19 is, thus, a public health emergency that is posing a challenge to emotional resilience and leading to psychological ramifications. ${ }^{1,2}$ The study findings from China, during the outbreak of COVID-19, highlighted that $53.8 \%$ of participants reported stress, anxiety, and depression. ${ }^{2}$ Moreover, the study reported that three-quarters of the participantswere worried about their family contracting the virus. ${ }^{2}$

Correspondence to: Dr. Sonia Ijaz Haider, Department for Educational Development, The Aga Khan University,

Stadium Road, Karachi, Pakistan

E-mail: sonia.i.haider@gmail.com

Received: May 21, 2020; Revised: June 27, 2020;

Accepted: June 30, 2020

DOI: https://doi.org/10.29271/jcpsp.2020.JCPSPCR.CR59
It is predicted that there will be negative mental health implications, which will last long after the pandemic has ended. Despite the fact that international agencies and other national, and local agencies are constantly attempting to provide accurate and frequent information about the disease, the influx of continuous information, misinformation, or lack of necessary information is a major problem.

Pakistan is the sixth most populous country in the world ${ }^{1}$ with a total population of approximately 220 million; among these, over 24 million are suffering from some form of mental disorder. ${ }^{3}$ During the recent COVID-19 outbreak, the existing mental health patients are faced with difficulties in receiving maintenance treatment, which has precipitated relapse, and led to uncontrollable behaviours and self-harm, and some form of mental illness. Besides, lack of education, neglect, and stigma regarding mental health conditions also compounded the existing burden. Thereisonly one psychiatrist available for 500,000 people, which is one of the lowest in the world. Being a developing country, it spends only $1 \%$ of its annual budget in the healthcare sector, with only $0.4 \%$ allocated for mental health needs.

The current COVID situation and the compounding effects have added to the existing psycho-social burden and; hence, have given rise to many important queries. 
What makes people panic amid the COVID-19 pandemic?

How mandatory lockdown has impacted the psycho-social wellbeing of people?

How the COVID-19 containment measures have led to risky behaviours?

\section{Why are people afraid of the quarantine?}

How the pandemic has worsened the existing mental illness symptoms?

What are some of the initiatives that have been undertaken to overcome mental distress and to promote psycho-social wellbeing amid COVID-19?

Literature suggest that the past pandemics of Ebola, H1N1, and SARS aggravated xenophobia, severe anxiety, PTSD, and disease-associated stigma. ${ }^{4}$ Similarly, in Pakistan, the outbreak of COVID-19 has caused panic and instilled fear, stress, and anxiety in people. ${ }^{1}$ These reactions could be due to several reasons; suspected isolated cases may suffer from anxiety due to uncertainty about their health condition, fear of death, the city of the virus, its infectivity, lack of definitive vaccination, and treatment and fragile healthcare systems with limited necessary facilities to screen and treat the infected cases. ${ }^{1,2}$ Lack of clarity and uncertainty about the meaning and extent of quarantine and lockdown further precipitate anxiety among the general public. Moreover, approximately one quarter of Pakistani population lives below the poverty line, and depends on daily wages to meet their needs. ${ }^{5}$ These people are now suffering more due to restrictions imposed by lockdown. ${ }^{1}$ The effects of pandemic on economic activities and the crash of stock markets have also added to apprehension and distress among people. ${ }^{6}$ Furthermore, insecurity about availability of basic items of daily living has resulted in hoarding of large volumes of food supplies, sanitizers, masks, tissue rolls, and other necessities, resulting in shortage of supplies for others. Consequently, the overall gravity of the situation has contributed to feelings of insecurity, xenophobia, fear, hate, exclusion, and rise in violence among the general population.

In the current situation, where neither vaccine nor definitive cure is available, social distancing and lockdowns are considered the most viable and practical preventive measures. While this strategy has worked effectively in containing the spread of the disease, particularly in China, ${ }^{2}$ the mandatory lockdown has adversely impacted the lives of people in numerous ways. Social habits (hugging, handshaking), physical activities, participation in religious gatherings, work engagement, and socialising with friends and families contribute significantly towards the psycho-social wellbeing of people. As these are linked with psychological wellness; this, in turn, acts as a buffer against stress or mental illness. The current scenario has changed the way of life and all these activities are prohibited now. Consequently, this social distancing has hit us in two ways - it has badly affected our daily routine of life, resulting in stress and anxiety, and it has taken away the channels that could help us cope in such situations, like physical activity, engagement, and connectedness.

Although, in current circumstances, virtual networking is encouraged, but, this, by no means can substitute physical connectedness. Moreover, the unpredictability regarding the duration of isolation can lead to indulgement in maladaptive behaviours, such as: overeating, physical inactivity, smoking, substance abuse, overuse of social media, domestic violence, insomnia, anger, non-compliance with the prescribed medical care, and suicidal/homicidal behaviours. ${ }^{1,5}$ Hence, people are facing challenges in dealing with the psycho-social impact of this crisis.

In Pakistan, the psychological impact is massive; and because of raised concerns about job insecurity and fear of getting disease, it is contributing to uncertainty, anger, insomnia and hopelessness. ${ }^{1}$ There has been corresponding increase in domestic violence, child abuse, as well as helplessness, and inclination towards suicidal tendencies. ${ }^{5}$

Quarantine has also generated hopelessness, apprehension and fear among people. ${ }^{1,2}$ Those who are unwell are reluctant to get screened because of the fear of isolation, along with the uncertainty about recovery. This fear is further compounded by the thought of not being able to meet their close ones and of dying alone without proper religious burial rituals. Moreover, people who have witnessed death of their beloved ones in the current pandemic, are likely to suffer from a high degree of guilt, resentment, anxiety or depression.

Stigma is another distressing factor that individuals, as well as the community at large, are challenged with. In Pakistan, COVID-19 is associated with similar stigmatisation and this fear prevents patients from going for screening and acquiring the required care.

It has also been reported that COVID-19 has aggravated the situation of those with pre-existing mental illnesses and has added to the suffering of the affected individuals. ${ }^{6}$ This deterioration is due to combination of overall fear, leading to anxiety and high stress levels. Closure of out-patient and other rehabilitation services, due to the lockdown, has led to disruption in their functional daily routine. Management of psychiatric patients is based on bio-psychosocial model; and due to the current situation, most of their prescribed management is interrupted. Engagement in occupational therapy activities (e.g. sports, socialisation, art therapy, and counselling sessions) is an important component and lack of it is resulting in a relapse of their symptoms. Although individuals with mental health issues are at risk in general, but specifically, people suffering from obsessive-compulsive disorders (OCD) are experiencing a relapse of their symptoms, due to continuous advice regarding hand washing, utilisation of disinfectants and care due to COVID-19. Additionally, people suffering from paranoia or having hypochondriasis, in particular, may become victims of undue fear and suspicion of contracting the disease. ${ }^{6}$ 
In mass emergencies, addressing psychological issues becomes a major health challenge. Several strategies have been adopted by different countries to overcome the psychological repercussions of COVID-19 outbreak. Compliance with WHO and governmental standard operating procedures (SOPs), like wearing a mask, maintaining social distances, and frequent hand-washing measures, provide a sense of safety and security and reduce psycho-social distress among the general population. ${ }^{2,5}$ Moreover, other strategies for minimising mental stress recommended by WHO include: refraining from stigmatising suspected or confirmed individuals and their families, limiting time to seek COVID-19 related information, following a routine, and listening or watching positive accounts of people who have recovered from the infection.

In order to cater to the mental health needs of its people during the outbreak, China has expanded their tele-health services and also implemented telephonic cognitive behavioural therapy (CBT), behavioural therapy, and relaxation exercises to improve the resilience of people during the lockdown. ${ }^{2}$

In Pakistan, the effects of the outbreak of COVID-19 on people's mental health status are well recognised. ${ }^{1,5}$ The country very soon realised the need of structured mental health services, considering the extent of psycho-social challenges due to the coronavirus outbreak. ${ }^{1}$ Hence, a helpline waslaunched for those in distress during the lockdown, called the mental health telemedicine helpline. Government is supported by local non-governmental agencies (NGOs) such as, Umang, Taskeen, Aman Foundation, Persukoon Zindagi, which have already been providing mental health support to the general public and are now even more supportive during this crisis.

Moreover, governmental agencies have made efforts to ensure the availability of masks, hand sanitizers, and soaps; particularly at religious places, business centres, shops, workplaces, and on public transport, to reduce the fear and anxiety associated with contracting the infection, among people.

Pakistan is in dire need of structured mental health activities to limit the burden of psychosocial challenges of the outbreak. ${ }^{1,5}$ The Government of Pakistan is constantly updating authentic information to provide psychosocial support to the public through its official website COVID-19 health advisory at http://covid.gov.pk/. The website entails the details of actions taken by the government to relieve anxiety and reduce phobia.

The Prime Minister's “Ehsaas” emergency cash programme has been launched to provide economic, mental and social relief to the vulnerable and needy. These measures will hopefully promote compliance with governmental lockdown regulations and provide mental relief to the community at large.

In addition, the government has also notified an anti-hoarding ordinance to address the issue of food insecurity, resulting from lockdown, which is linked with the mental health status of public. "Ehsaas" is supported by several commercial, and non-commercial agencies to provide food (ration, langar) and essential supplies to the vulnerable segments of the society. Other initiatives, such as shelter (Panahgah App) have also been launched. In compliance with lockdown strategies, the radio programme "Raabta" is being used for payment notifications to all applicants registered for "Ehsaas Emergency Cash".

Pakistan Television Corporation has also initiated online tele-school courses for students of grades 1-12 to engage them in the regular education program. Not only students, but teachers are also benefiting from this programme and distracting themselves from the hazards of unchecked social media information on COVID-19. Other organisations, mainly NGOs, have also taken initiatives to improve the psychological wellbeing of the population during COVID-19 pandemic. The Aga Khan Foundation (AKF) has published a document in multiple languages, named "Guidance on psychosocial wellbeing and support during COVID-19", for individuals, families, and HCPs. Agency Standing Committee (IASC) has published a guide for COVID-19 responders, called "basic psychosocial skills". Anothernon-governmental organisation, Sehat Kahani, has launched a mental health response website, in collaboration with local and international partners, to address the increasing mental health burden.

Many private hospitals and institutions have also come forward. At Aga Khan University Hospital, tele-clinics, and helpline have been started in different specialities, including psychiatry. This is a relatively new approach that has been adopted in several healthcare facilities in the country, and is appreciated by patients who require health consultation, but are scared of going to hospitals and clinics for fear of being infected by the disease.

The print and electronic media have also been playing an important role since the beginning of the pandemic in the country. The media is not only clarifying the prevailing misconceptions, but is also educating people about the stigma associated with COVID-19. Awareness campaigns in local languages are being broadcast using all available options, like morning shows, online doctor consultations, animated short communication messages, messages from influential celebrities, and advertisements. Through these means, they are conveying the strategies that need to be adopted, both to prevent the infection and to control its spread to others, and giving information about medical care and home management during the quarantine and isolation periods, to address people's physical and mental health concerns.

The social media platforms, such as Facebook, Youtube, and several others are also being extensively used to promote healthy lifestyles and the adoption of coping mechanisms during the lockdown period. Moreover, to minimise the negative impact of the lockdown, online modalities and virtual modes of engaging in education, work, and socialisation with friends, and families are being regularly conveyed to the people. These platforms are also constantly providing various activities that people can undertake for a healthy lifestyle, like sports, yoga, music, arts, and mindfulness.

Due to a shortage of mental health experts in the country, there 
is a dire need to train healthcare providers with basic psychological first-aid training, so as to equip them to provide holistic care. Moreover, a qualified mental health professional should be made a part of the COVID-19 taskforce group, to ensure a holistic approach. Furthermore, capacity building of taskforce volunteers, teachers, workplace staff, religious and political leaders, with regard to psycho-social management skills, is needed, as this will be a valuable asset for screening and counselling of mental health problems. Likewise, psychosocial support services should be provided for at least three to six months, in the post-isolation period, for vulnerable populations. This support should encompass mental health facilities from screening to counselling, along with the provision of the basic food supply.

The medical curriculum should be geared towards the management of trauma arising out of public health emergencies, particularly those which can translate into psychiatric disorders, such as: anxiety, depression, and suicidal behaviours. Moreover, prospective studies targeting the quality of life of those in quarantine and in isolation centres must be carried out. Furthermore, the isolation centres must be equipped with telephonic services which should ensure patients' daily communication with close family members. Additionally, the government should also take measures to ensure that individuals' religious and cultural values are protected. The government should also conduct research studies in collaboration with international partners to understand the new infection and strategies that have been implemented and have been tested effectively to reduce the psychosocial distress and improve mental health wellbeing.

History tells us that all the pandemics were time-bound and eventually ended one day. Hence, the temporality of the current pandemic must be reinforced to promote hope and a futuristic outlook in people. Public health interventions should mitigate and address stigma and psycho-social issues by actively engaging communities and relevant stakeholders. This pandemic calls for a unified global approach to eradicate the suffering of the common people, which is a goal that lies at the centre of the global agenda of "health for all". This will be possible only when the government will give due priority to the mental health domain, particularly during this COVID-19 crisis

\section{CONFLICT OF INTEREST:}

Authors declared no conflict of interest

\section{AUTHORS' CONTRIBUTION:}

SF: Conception and design, drafting, final approval.

SS: Conception and design, critical revision, final approval.

$\mathrm{SIH}$ : Design, interpretation, critical revision, final approval.

SI, YP: Interpretation, critical revision, final approval.

HS: Critical revision, final approval.

\section{REFERENCES}

1. Mukhtar S. Preparedness and proactive infection control measures of Pakistan during COVID-19 pandemic outbreak. Res Social Adm Pharm 2020; S1551-7411(20)30373-9.

2. Wang C, Pan R, Wan X, Tan Y, Xu L, Ho CS, et al. Immediate psychological responses and associated factors during the initial stage of the 2019 coronavirus disease (COVID-19) epidemic among the general population in China. Int J Environ Res Public Health 2020; 17(5):1729.

3. WHO. Depression and other common mental disorders: global health estimates. World Health Organization, 2017. http://apps.who.int/iris/bitstream/handle/10665/254610/WH O-MSD-MER-2017.2-eng.pdf

4. Prati G, Pietrantoni L. Knowledge, risk perceptions, and xenophobic attitudes: evidence from italy during the ebola outbreak. Risk Anal 2016; 36(10):2000-10.

5. Mamun MA, Ullah I. COVID-19 suicides in Pakistan, dying off not COVID-19 fear but poverty? - The forthcoming economic challenges for a developing country. Brain Behav Immun 2020; 87:163-6.

6. Rajkumar RP. COVID-19 and mental health: A review of the existing literature. Asian J Psychiatr 2020; 52:102066. 